\title{
General Hubbard model for strongly interacting fermions in an optical lattice and its phase detection
}

\author{
L.-M. DUAN
}

FOCUS center and MCTP, Department of Physics, University of Michigan - Ann Arbor, MI 48109, USA

received 18 September 2007; accepted 6 November 2007

published online 3 December 2007

PACS 03.75.Ss - Degenerate Fermi gases

PACS 05.30.Fk - Fermion systems and electron gas

PACS 34.50.-s - Scattering of atoms and molecules

\begin{abstract}
Based on the consideration of the system symmetry and its Hilbert space, we show that strongly interacting fermions near a wide Feshbach resonance in an optical lattice or superlattice can be generically described by a lattice resonance Hamiltonian. The latter can be mapped to a general Hubbard model with particle-assisted tunnelling rates. We investigate the model under population imbalance and show that the attractive and the repulsive models have the same complexity in the phase diagram under the particle-hole mapping. Using this mapping, we propose an experimental method to detect possible exotic superfluid/magnetic phases for this system.
\end{abstract}

Copyright (c) EPLA, 2008

Among the control techniques for ultracold atoms, optical lattice and Feshbach resonance play particularly important roles. The optical lattice is used to control the interaction configuration while the Feshbach resonance is a tool to tune the interaction magnitude. The combination of these two powerful techniques naturally becomes the next frontier, which has attracted significant recent interest $[1-5]$. To understand this important system, one needs to have a Hamiltonian to describe strongly interacting atoms in an optical lattice. The starting Hamiltonian is unfortunately complicated as one has to take into account multi-band populations as well as direct neighboring couplings $[2,4,5]$. We have described a method in [5] to derive an effective lattice Hamiltonian for this system from the field theory of the two-channel model.

In this paper, we report the following advance along this direction: firstly, based on the consideration of the system symmetry and its Hilbert space, we show that a lattice resonance model turns out to be a generic Hamiltonian for this system. The resulting Hamiltonian agrees with the one from our previous microscopic derivation [5], but the method used here shows this Hamiltonian should have general applicability. As an example, we point out that for strongly interacting fermions in optical superlattices, the effective Hamiltonian is again described by this lattice resonance model when we introduce some dressed degrees of freedom. For certain configurations of the superlattice, the system naturally supports a $d$-wave superfluid. Secondly, we mathematically map the lattice resonance Hamiltonian to a general Hubbard model (GHM) with particle-assisted tunnelling rates. The particle-assisted tunnelling brings in some new feature, in particular, it may favor a superfluid phase compared with the Hubbard model. Thirdly, we discuss the attractive Hubbard model with population imbalance between the two spin components, and show it has the same complexity in the phase diagram as the repulsive Hubbard model under a particle-hole mapping. This result is related to the recent large effort to understand the polarized Fermi gas $[6,7]$. Finally, using the mapping above, we propose an experimental scheme to detect possible exotic superfluid or magnetic orders in this system. The method is based on Raman-pulse-assisted time-of-flight imaging, and can reveal the superfluid or magnetic phases with detailed information about the order parameter or the pairing wave function.

For strongly interacting two-component (effectively spin-1/2) fermions in an optical lattice, when two atoms with different spins come to the same site, they form a dressed molecule with atomic population distributed over many lattice bands due to the strong on-site interaction $[4,5]$. We consider the system with an average atom filling number $\bar{n} \leqslant 2$. In this case, the possibility of the 3 -atom occupation of a single site is negligible as that is suppressed at low temperature by an energy cost about the lattice band gap [8]. We then have only four possible configurations for each site $i$, either empty, or a spin$\sigma(\sigma=\uparrow, \downarrow)$ atom, or a dressed molecule (the structure 

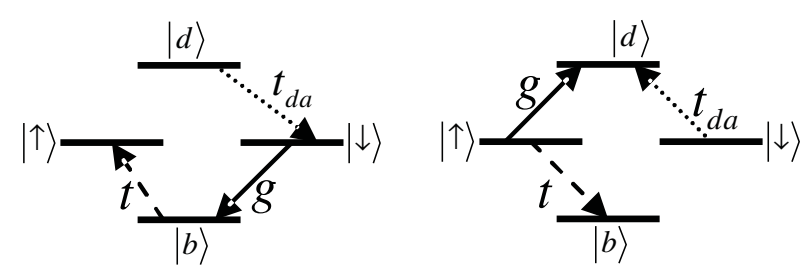

Fig. 1: Illustration of the configuration tunnelling between two neighboring sites. The process shown in the figure correspond to the $t, g$, and $t_{d a}$ terms in the Hamiltonian.

of the dressed molecule fixed by the single-site two-body physics includes the multi-band population effects). The creation operators for these configurations are denoted by $b_{i}^{\dagger}, a_{i \sigma}^{\dagger}, d_{i}^{\dagger}$, respectively, while the corresponding states are written as $|b\rangle_{i},|\uparrow, \downarrow\rangle_{i},|d\rangle_{i}$. We introduce the slave boson operator $b_{i}^{\dagger}$ for creation of an empty site $i$ so that the constraint of the Hilbert space on each site can be simply implemented through

$$
b_{i}^{\dagger} b_{i}+a_{i \uparrow}^{\dagger} a_{i \uparrow}+a_{i \downarrow}^{\dagger} a_{i \downarrow}+d_{i}^{\dagger} d_{i}=I .
$$

Note that with this constraint $a_{i \sigma}$ describe fermions, while $d_{i}$ and $b_{i}$ both represent hard-core bosons.

We assume the system has a global $S U(2)$ symmetry for the spin components. In that case, $|\uparrow\rangle_{i}$ and $|\downarrow\rangle_{i}$ are degenerate in energy, and the most general form of the single-site Hamiltonian can be written as $H_{i}=$ $-\mu \sum_{\sigma} a_{i \sigma}^{\dagger} a_{i \sigma}+(\Delta-2 \mu) d_{i}^{\dagger} d_{i}$, where we have absorbed the single-atom energy into the definition of the chemical potential $\mu$, and $\Delta$ is the relative energy shift of the dressed molecule. For two neighboring sites $i$ and $j$, due to the atomic tunnelling and off-site interactions, there will be a Hamiltonian term $H_{i j}$ to describe all the possible configuration tunnelling or couplings. With the spin $S U(2)$ symmetry and the number conservation of each spin component, the most general two-site Hamiltonian can be written as $H_{i j}=H_{i j}^{(1)}+H_{i j}^{(2)}$, where $H_{i j}^{(1)}$ describes the configuration tunnelling that involves transfer of one atom with the following form (see the illustration in fig. 1):

$$
\begin{aligned}
H_{i j}^{(1)}= & \sum_{\sigma}\left(t a_{i \sigma}^{\dagger} b_{i} b_{j}^{\dagger} a_{j \sigma}+t_{d a} d_{i}^{\dagger} a_{i \sigma} a_{j \sigma}^{\dagger} d_{j}\right) \\
& +g\left(d_{i}^{\dagger} b_{j}+d_{j}^{\dagger} b_{i}\right)\left(a_{i \uparrow} a_{j \downarrow}-a_{i \downarrow} a_{j \uparrow}\right)+\text { H.c. },
\end{aligned}
$$

and $H_{i j}^{(2)}$ describes the configuration coupling that involves real or virtual tunnelling of two atoms with the general expression

$$
\begin{aligned}
H_{i j}^{(2)}= & \left(t_{d} d_{i}^{\dagger} b_{i} b_{j}^{\dagger} d_{j}+\text { H.c. }\right)+x_{d} n_{d i} n_{d j} \\
& +x_{a} n_{i} n_{j}+x_{s} \mathbf{s}_{i} \cdot \mathbf{s}_{j}+x_{b} n_{b i} n_{b j} .
\end{aligned}
$$

In $H_{i j}^{(2)}$, the number and the spin operators are defined by $n_{d i} \equiv d_{i}^{\dagger} d_{i}, n_{i} \equiv a_{i \uparrow}^{\dagger} a_{i \uparrow}+a_{i \downarrow}^{\dagger} a_{i \downarrow}, \quad n_{b i} \equiv b_{i}^{\dagger} b_{i}$, and $\mathbf{s}_{i} \equiv$ $\sum_{\sigma \sigma^{\prime}} a_{i \sigma}^{\dagger} \sigma_{\sigma \sigma^{\prime}} a_{i \sigma^{\prime}} / 2$ ( $\sigma_{\sigma \sigma^{\prime}}$ is the Pauli matrix). The term $n_{b i} n_{b j}$ is equivalent to the cross coupling $n_{d i} n_{j}+n_{i} n_{d j}$ under the constraint (1). By analyzing the level configurations in fig. 1 , one can convince oneself that $H_{i j}^{(1)}$ and $H_{i j}^{(2)}$ include all the possible two-site coupling terms with the $S U(2)$ symmetry. As the atomic interactions are short range, all the multiple site couplings can be neglected. So a generic lattice Hamiltonian is given by $H=$ $\sum_{i} H_{i}+\sum_{\langle i, j\rangle}\left(H_{i j}^{(1)}+H_{i j}^{(2)}\right)$, where $\langle i, j\rangle$ denotes neighboring sites. This Hamiltonian describes the coupling between the fermionic atoms $a_{i \sigma}$ and the bosonic dressed molecules $d_{i}$ with a detuning $\Delta$, and will be referred in the following as the lattice resonance model.

The Hamiltonian $H$, together with the constraint (1), poses a well-defined problem. Note that $H$ agrees in form with the effective lattice Hamiltonian for strongly interacting fermions that we derived before from a completely different method [5]. The only specification from that microscopic derivation is to fix the coefficients $x_{b}=0$ and $x_{a}=-x_{s} / 4$. As mentioned in [5], in the case of a large detuning $\Delta$, the Hamiltonian $H$ is reduced to either the $t$ - $J$ model for atoms or the XXZ model for dressed molecules, depending on which species get populated. We also notice that for short-range interactions, with increase of the lattice potential barrier, all the interaction coefficients in $H_{i j}^{(2)}$ decay much faster compared with those in $H_{i j}^{(1)}$. So for a lattice with sufficient depth, $H_{i j}^{(1)}$, dominates over $H_{i j}^{(2)}$, and in the following, without special mention we will consider the simplified Hamiltonian $H=\sum_{i} H_{i}+\sum_{\langle i, j\rangle} H_{i j}^{(1)}$ by dropping $H_{i j}^{(2)}$.

We now recast the Hamiltonian $H$ into a different form which shows its connection with the Hubbard model. For this purpose, we map the dressed molecule state $d_{i}^{\dagger}|v a c\rangle$ to the two-fermion state $a_{i \downarrow}^{\dagger} a_{i \uparrow}^{\dagger}|v a c\rangle$, where $|v a c\rangle$ denotes the vacuum. Note that physically the structure of the dressed molecules should be determined by diagonalizing the on-site interaction Hamiltonian, and it generally involves superposition of atoms in many-band configurations [4,5], which is certainly different from the state $a_{i \uparrow}^{\dagger} a_{i \downarrow}^{\dagger}|v a c\rangle$ with double occupation on a single band. But mathematically we can identify these two states by a one-to-one mapping. After this mapping, the Hamiltonian $H$ can be written in the form

$$
\begin{aligned}
H= & \sum_{i}\left[(\Delta / 2) n_{i}\left(n_{i}-1\right)-\mu n_{i}\right] \\
& +\sum_{\langle i, j\rangle, \sigma}\left[t+\delta g\left(n_{i \bar{\sigma}}+n_{j \bar{\sigma}}\right)+\delta t n_{i \bar{\sigma}} n_{j \bar{\sigma}}\right] a_{i \sigma}^{\dagger} a_{j \sigma}+\text { H.c. }
\end{aligned}
$$

where $\quad \delta g \equiv g-t, \quad \delta t \equiv t_{d a}+t-2 g \quad$ and $\quad n_{i \bar{\sigma}} \equiv a_{i \bar{\sigma}}^{\dagger} a_{i \bar{\sigma}}$ $(\bar{\sigma}=\downarrow, \uparrow$ for $\sigma=\uparrow, \downarrow)$. To verify that the two forms of $H$ in eqs. (2) and (4) are equivalent to each other, one can check the physical process represented by each term to confirm it is identical. Note that in this new form of $H$, there is no need of the slave boson operator to constraint the Hilbert space as the latter is automatically fixed by the properties of fermions. As there is no additional constraint, the Hamiltonian in the form of eq. (4) looks simpler and may be easier for treatment in certain cases. Compared with the conventional Hubbard model, the effective tunnelling 

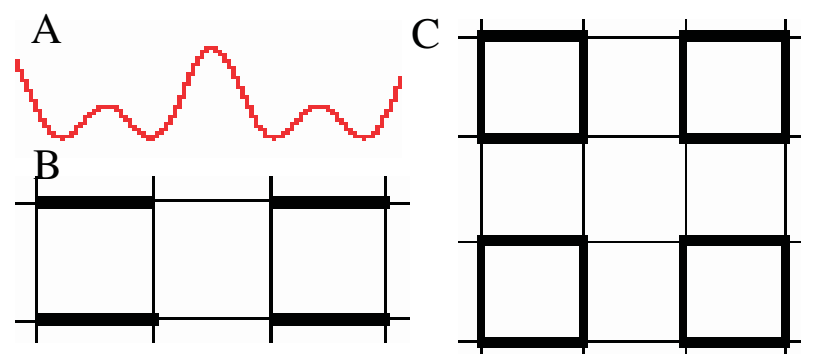

Fig. 2: Illustration of an optical superlattice: (A) the superlattice potential. (B,C) The dimer and the plaquette lattices (bold lines represent stronger coupling) formed with the potential in $(\mathrm{A})$.

rate in $H$ becomes an operator which depends on the occupation of the two sites. The original lattice resonance Hamiltonian in eq. (2) is thus mapped to a general Hubbard model (GHM) with particle-assisted tunnelling rates. For weakly interacting fermions, the multi-band population and the direct neighboring coupling become negligible, then the coefficients $g$ and $t_{d a}$ tend to $t_{a}$, and the GHM returns to the conventional Hubbard model as one expects in this case [9].

The derivation of the Hamiltonian $H$ in this work is based on very general arguments about the single-site Hilbert space and the system symmetry. This reminds us that $H$ has a generic form which should apply to different systems with similar Hilbert space structure and symmetry properties. As an example, we point out that for interacting fermions in an optical superlattice, under several interesting configurations, the system is also well described by the above Hamiltonian $H$. Figure $2 \mathrm{~A}$ illustrates an optical superlattice potential which can be realized with two standing-wave laser beams [10]. With a combination of this superlattice and the conventional optical lattice potentials, one can realize the dimer or plaquette lattices as illustrated in figs. $2 \mathrm{~B}$ and $\mathrm{C}$ where the intra-dimer (intra-plaquette) couplings are much stronger than the inter-dimer (inter-plaquette) couplings. To derive an effective Hamiltonian for this system, one needs to first construct dress energy levels for each dimer (plaquette) by exactly solving a few-site problem. For twocomponent interacting fermions in those lattices near half filling, the low-energy level configurations from each dimer (plaquette) have basically the same structure as those shown in fig. $1[11,12]^{1}$, and the system also has the $S U(2)$ symmetry. We then immediately conclude that the Hamiltonian in the forms of eq. (2) or (4) should be applicable to describe physics in the dimer or plaquette lattices around half filling. The two-dimensional plaquette lattice is particularly interesting: because of the internal plaquette structure, the excitation from $|b\rangle_{i}$ to $|d\rangle_{i}$ states in eq. (2) has a $d$-wave symmetry (e.g., $\left\langle d_{i}^{\dagger} b_{i}\right\rangle$ flips

\footnotetext{
${ }^{1}$ For a plaquette configuration, the levels $|\uparrow\rangle$ and $|\downarrow\rangle$ are actually doubly degenerate due to the rotational symmetry (see $[11,12]$ ), but the physical picture is basically the same by adding a pseudospin index for this degeneracy.
}

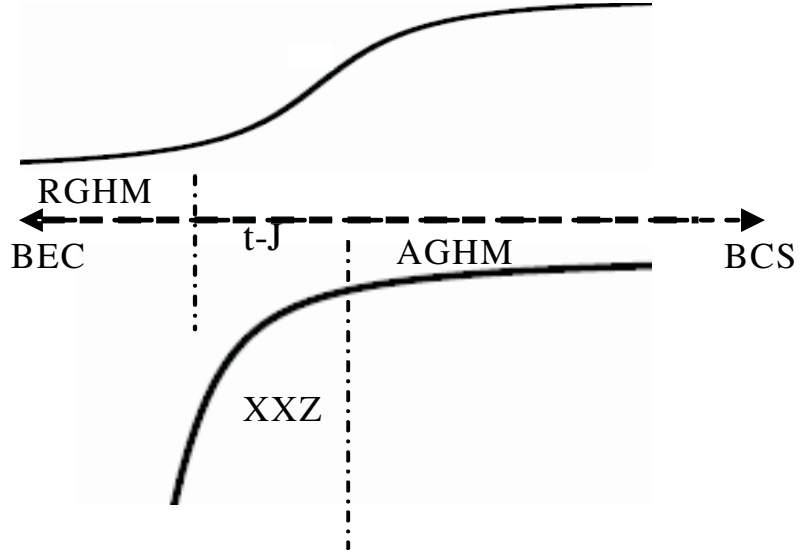

Fig. 3: The effective Hamiltonians in different regions. The solid curves correspond to two dressed molecule bands, and the middle dashed line is an atomic band. On the BCS or BEC (with population mainly in atoms) sides, the Hamiltonians are given by the attractive (repulsive) general Hubbard models (AGHM and RGHM), respectively. As one increases the detuning $\Delta$, one gets either the XXZ model for the dressed molecules or the $t-J$ model for the atoms. In the deep BCS or BEC limit, the GHM returns to the attractive or repulsive Hubbard model, and the XXZ Hamiltonian yields to the bosonic Hubbard model for molecules when multiple occupation of a single site is allowed by weaker effective interaction.

sign under a $\pi / 2$ rotation of the lattice) $[11,12]$. When the effective detuning $\Delta$ is tuned to be negative, one can show under pretty well-controlled approximations that the plaquette lattice in this configuration supports the $d$-wave superfluid $[11,12]$.

We now investigate some properties of the GHM in eq. (4). When the detuning $\Delta$ is negative, similar to the Hubbard model, we expect this Hamiltonian is in a superfluid state away from the unit filling. When $\Delta$ is positive, although it is not clear yet whether $H$ has a superfluid state, compared with the corresponding repulsive Hubbard model, we do expect that the superfluid possibility becomes higher when $g>t_{a}$ (which is very likely the case for fermionic atoms near a wide Feshbach resonance [5]) as a large- $g$ term (see eq. (2)) clearly favors Cooper pairing. From the single-site physics, we know that two atoms always have an on-site bound state (corresponding to a negative $\Delta$ ) with the binding energy $(-\Delta)$ approaching zero as one moves to the BCS side of the Feshbach resonance [4,5]. So in the ground-state configuration, the strongly interacting Fermi gas naturally implement the GHM with a negative $\Delta$. To experimentally investigate the GHM with a positive $\Delta$, one needs to start with the population in atoms (instead of Feshbach molecules), and to approach the Feshbach resonance from the BEC side (the system is in a metastable state in this case). The effective Hamiltonians in different regions are shown in fig. 3 .

When we take into account possible population imbalance between the two spin components, the repulsive and 
the attractive GHMs (with positive or negative $\Delta$, respectively) become intrinsically connected, and they should have the same complexity in phase diagram. Polarized Fermi gas recently raised a lot of interest [6], and in free space (or in a weak trap), although population imbalance yields some new features, the basic physics there is still largely captured by an extension of BCS type of meanfield theory [7]. However, for polarized Fermi gas in an optical lattice, we show that simple extensions of the BCS theory are very likely to give misleading results because of the exact mapping between the repulsive and the negative GHMs. Population imbalance corresponds to introduction of an effective magnetic field $h$, which adds a term $-h \sum_{i} \sigma_{i}^{z}\left(\sigma_{i}^{z} \equiv n_{i \uparrow}-n_{i \downarrow}\right)$ to the Hamiltonian $H$ in eq. (4). We apply a particle-hole transformation $a_{i \uparrow} \rightarrow a_{i \uparrow}$ and $a_{i \downarrow} \rightarrow(-1)^{i} a_{i \downarrow}^{\dagger}$ to the Hamiltonian [13] (for simplicity, we consider a bi-partite lattice). Under this transformation, the Hamiltonian $H-h \sum_{i} \sigma_{i}^{z}$ is mapped to

$$
\begin{aligned}
H^{\prime}= & \sum_{i}\left[-(\Delta / 2) n_{i}\left(n_{i}-1\right)-\mu^{\prime} \sigma_{i}^{z}-h^{\prime} n_{i}\right] \\
& +\sum_{\langle i, j\rangle, \sigma}\left[t_{\sigma}+\delta g_{\sigma}\left(n_{i \bar{\sigma}}+n_{j \bar{\sigma}}\right)+\delta t n_{i \bar{\sigma}} n_{j \bar{\sigma}}\right] a_{i \sigma}^{\dagger} a_{j \sigma}+\text { H.c. }
\end{aligned}
$$

where the parameters $\mu^{\prime} \equiv \mu-\Delta / 2, h^{\prime} \equiv h-\Delta / 2, t_{\uparrow} \equiv t$, $t_{\downarrow} \equiv t_{d a}, \quad \delta g_{\sigma} \equiv g-t_{\sigma}, \quad$ and we neglect the constant energy per site $h-\mu$. One can see that an attractive GHM $(\Delta<0)$ is mapped exactly to a dual repulsive model (with $-\Delta$ ), where the chemical potential $\mu$ and the filed $h$ exchange their roles. Superfluid phases (including both the BCS state and the LOFF (Larkin-Ovchinnikov-FuldeFerrel) state with pairing at nonzero momenta [14]) of the original model correspond to magnetic phases of the dual model and vice versa. For a repulsive Hubbard model on a square lattice, the magnetic order exists only in a region near half filling, and with hole doping there are possibilities of exotic phases including a non-BCS superfluid state. This suggests that for the attractive Hubbard model with population imbalance, the superfluid phase exists in the region with small polarization. With further increase of the polarization, there could appear exotic phases including a $d$-wave magnetic order. Experimental investigation of the attractive GHM with population imbalance (which might be easier for realization compared with the repulsive one) is therefore able to provide critical information to understand the challenging phase diagram of the repulsive Hubbard model.

Finally, we propose a method to detect possible exotic phases in this system by making use of the above mapping. Our purpose is to directly measure the magnetic or superfluid order parameters. The detection scheme combines the time-of-flight imaging with some instantaneous Raman pulses [15]. We take ${ }^{6} \mathrm{Li}$ atoms as a typical example. The scheme is illustrated in fig. 4. Right after turn-off of the trap, we immediately apply two consecutive impulsive Raman pulses. These pulses are assumed faster than the

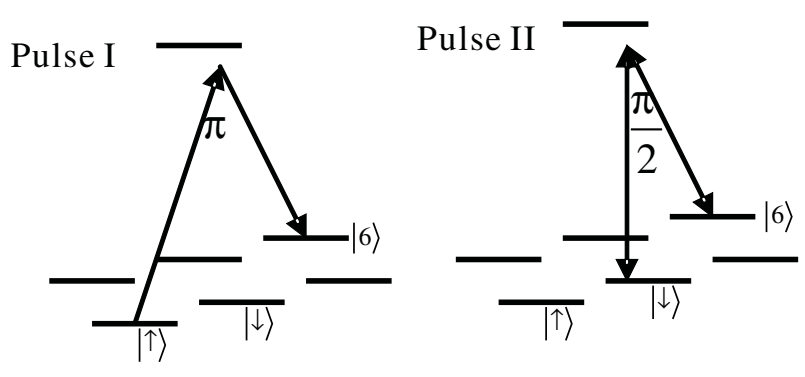

Fig. 4: Illustration of the two Raman pulse (a $\pi$ and a $\pi / 2$ pulse, respectively) before the time-of-flight for measuring the correlation function in eq. (6). We use the level structure of ${ }^{6} \mathrm{Li}$ atoms as an example (with the magnetic field near the wide Feshbach resonance).

system dynamics (characterized by the Fermi energy), but slower compared with the level splitting between the $|\uparrow\rangle$ and $|\downarrow\rangle$ levels (about $70 \mathrm{MHz}$ ). The first is a $\pi$-pulse, consisting of two laser beams propagating along different directions, which transfers the atoms from the level $|\uparrow\rangle$ to $|6\rangle$ by imprinting a photon recoil momentum -q. As the level $|6\rangle$ is detuned from $|\uparrow\rangle$ by a few $\mathrm{GHz}$, this transition at the same time tune the system out of the Feshbach resonance (the atoms in states $|6\rangle$ and $|\downarrow\rangle$ are only weakly interacting) [15]. The second is a $\pi / 2$ pulse from co-propagating laser beams applied to the levels $|6\rangle$ and $|\downarrow\rangle$, which induces a transformation $a_{\mathbf{k} 6} \rightarrow\left(a_{\mathbf{k} 6}+e^{i \varphi} a_{\mathbf{k} \downarrow}\right) / \sqrt{2}$ and $a_{\mathbf{k} \downarrow} \rightarrow\left(a_{\mathbf{k} \downarrow}-e^{-i \varphi} a_{\mathbf{k} 6}\right) / \sqrt{2}$ that preserve the momentum $\mathbf{k}$ ( $\varphi$ is the relative laser phase). After these two pulses, we take the time-of-flight images (with basically ballistic expansion) for the atoms in levels $|6\rangle$ and $|\downarrow\rangle$, and the difference of these two images give exactly the cross correlation of the $\uparrow$ and $\downarrow$ spin-components at different momenta:

$$
n_{\mathbf{k} 6}-n_{\mathbf{k} \downarrow}=2 \operatorname{Re}\left(e^{i \varphi} a_{\mathbf{k}+\mathbf{q}, \uparrow}^{\dagger} a_{\mathbf{k} \downarrow}\right) .
$$

We now show through a few examples that we can directly confirm various magnetic or superfluid phases with this detection ability. i) For magnetic phases with a pretty general form of the spin order parameter $\left\langle\mathbf{s}_{i}\right\rangle=$ $\mathbf{v}_{1} \cos \left(\mathbf{Q} \cdot \mathbf{r}_{i}\right)+\mathbf{v}_{2} \sin \left(\mathbf{Q} \cdot \mathbf{r}_{i}\right)$ [13], we can confirm it with sharp peaks for the correlation in eq. (6) when the relative momentum $\mathbf{q}$ is scanned to $\pm \mathbf{Q}$. The spin vectors $\mathbf{v}_{1}$ and $\mathbf{v}_{2}$ can be inferred from the relative laser phase $\varphi$. ii) For the LOFF superfluid state with pairing at a nonzero momentum $\mathbf{q}$, the order parameter $\left\langle a_{\mathbf{k}+\mathbf{q}, \uparrow} a_{-\mathbf{k} \downarrow}\right\rangle$ is nonzero. After the particle-hole mapping, this order parameter corresponds to a magnetic order $\left\langle a_{-\mathbf{k}-\mathbf{q}, \uparrow}^{\dagger} a_{-\mathbf{k} \downarrow}\right\rangle$ of the dual Hamiltonian. The peak of the correlation function in eq. (6) at the relative momentum $-\mathbf{q}$ thus confirms Bose condensation to a nonzero pair momentum for the original Hamiltonian, and the distribution in $\mathbf{k}$ of the correlation $\left\langle a_{-\mathbf{k}-\mathbf{q}, \uparrow}^{\dagger} a_{-\mathbf{k} \downarrow}\right\rangle$ gives the original pair wave function. iii) Similar to the LOFF state, for a $d$-wave superfluid phase with the order parameter $\left\langle a_{\mathbf{k}, \uparrow} a_{-\mathbf{k} \downarrow}\right\rangle \propto$ $\cos k_{x}-\cos k_{y}$, the pair wave function and its spatial 
symmetry can be directly measured by detecting the correlation (6) for the dual Hamiltonian.

In summary, we have established the results as we outlined in the introduction.

This work was supported by the MURI, the DARPA, the NSF award (0431476), the DTO under ARO contracts, and the A. P. Sloan Fellowship.

\section{REFERENCES}

[1] Chin J. K. et al., Nature (London), 443 (2006) 961.

[2] Stoeferle T. et al., Phys. Rev. Lett., 96 (2006) 030401.

[3] Dickerscheid D. B. M. et al., Phys. Rev. A, 71 (2005) 043604; Carr L. and Holland M., Phys. Rev. A, 72 (2005) 031604; Zhou F. and Wu C., New J. Phys, 8 (2006) 166.

[4] Diener R. B. and Ho T.-L., Phys. Rev. Lett, 96 (2006) 010402 .
[5] Duan L.-M., Phys. Rev. Lett., 95 (2005) 243202, condmat/0508745.

[6] Zwierlein M. W., Schirotzek A., Schunck C. H. and Ketterle W., Science, 311 (2006) 492; Partridge G. B. et al., Science, 311 (2006) 503.

[7] For a review, see Sheehy D. E. and Radzinovsky L., cond-mat/0607803.

[8] Kestner J. and DuAn L.-M., arXiv:0706.4123.

[9] Hofstetter W. et al., Phys. Rev. Lett., 89 (2002) 220407.

[10] Sebby-Strabley J., Anderlini M., Jessen P. S. and Porto J. V., cond-mat/0602103; Trebst S., Schollwoeck U., Troyer M. and Zoller P., Phys. Rev. Lett., 96 (2006) 250402.

[11] Tsai W.-F. and Kivelson S. A., Phys. Rev. B, 73 (2006) 214510.

[12] Goodman T. and Duan L.-M., Phys. Rev. A, 74 (2006) 052711; unpublished.

[13] Auerbach A., Interacting Electrons and Quantum Magnetism (Springer-Verlag, New York) 1994.

[14] For a review, see CASAlbuoni R. and NARDulli G., Rev. Mod. Phys., 76 (2004) 263.

[15] Duan L.-M., Phys. Rev. Lett., 96 (2006) 103201; Yi W. and Duan L.-M., Phys. Rev. Lett., 97 (2006) 120401. 